\title{
THE METHOD OF REGULATION IN BEHAVIOR AND IN OTHER FIELDS.
}

BY

H. S. JENNINGS.

The results set forth in the preceding paper, together with certain other relations found in the behavior of lower organisms, that have been detailed in previous papers by the present writer, suggest a certain point of view in regard to the general method of regulation or adjustment in organisms. Everywhere in the study of life processes we meet the puzzle of regulation. Organisms do those things which advance their welfare. There are some exceptions, but this is certainly true in a general survey. If the environment changes, the organism changes to meet the new conditions. If the mammal is heated from without, it cools from within; if it is cooled from without it heats from within, maintaining the temperature that is to its advantage. The dog which is fed a starchy diet produces digestive juices that are rich in the enzyms which digest starch, while under a diet of meat it produces juices rich in proteid digesting substances. When a poison is injected into a mouse, the mouse produces substances which neutralize this poison. If a part of the organism is injured, a rearrangement of material follows till the injury is repaired. If a part is removed, it is restored, or the wound is at least closed up and healed, so that the life processes may continue without disturbance. Regulation constitutes perhaps the greatest problem of biology. How can the organism thus provide for its own needs? To put the question crudely, how does it know what to do when a difficulty arises, so as to overcome this difficulty? It seems to work toward a definite purpose. In other words, the final result of its action seems to be present in some way at the beginning, determining what the action shall be. In this the action of living things appears to contrast with that of things inorganic. It is regulation of this character that has given us the theories of vitalism. By these theories the principles controlling the life 
processes are held to be of a character essentially different from anything found in the inorganic world.

Nowhere is regulation more striking than in the behavior of organisms. Indeed, the processes in this field have long served as the prototype for regulatory action. The organism moves and reacts, on the whole, in ways that are advantageous to it. If it gets into hot water, it takes measures to get out again, and the same is true if it gets into excessively cold water. If it enters an injurious chemical substance, it at once changes its behavior and escapes. If it lacks material for its metabolic processes, it sets in operation movements which secure such material, suspending these movements when the lack is fully supplied. If it lacks oxygen for respiration, it moves to a region where oxygen is found. If injured it flees to safer regions. In innumerable details it does those things which are good for it, and this is as true of the Protozoan as of the Metazoan. It is plain that behavior depends largely on the needs of the organism, and is of such a character as to satisfy these needs. In other words, behavior is adjustment or regulation.

There seems no reason to think that regulation in behavior is of a different character from that found elsewhere. But nowhere else is it possible to perceive so clearly how regulation occurs. In the behavior of the lowest organisms we can see not only what the animal does, but precisely how this happens to be regulatory. The method of regulation lies open before us. This method is of such a character as to suggest the possibility of its application to other fields; in other words, it suggests a possible general explanation of the method of regulation. This suggestion the present paper attempts to develop.

In the lower, unicellular, organisms where we can see just how regulation occurs, the process is as follows. Anything injurious to the organism causes changes in behavior. These changes subject the organism to new conditions. As long as the injurious condition continues, the changes in behavior continue. The first change in behavior may not be regulatory, nor the second, nor the third, nor the tenth. But if the changes continue, subjecting the organism successively to all possible different conditions, a condition will finally be reached that relieves the organism of the injurious action, provided such a condition exists. Thereupon the changes in behavior cease, and the organism remains in the 
favorable condition. The movements of the organism when stimulated are such as to subject it to various conditions, one of which is selected.

This method of regulation is found in its purest form in unicellular organisms, such as Paramœcium and Stentor. Yet it occurs also in higher organisms, and indeed is found in a less primitive form throughout the animal series, up to and including man. When we ourselves, or other animals, are confronted with a difficulty for which neither experience nor inherited tendency has furnished us with a direct method of relief, the only recourse is to this same method of regulation. We perform movements which subject us to various conditions, till one is found that relieves the difficulty. We call the process searching, testing, trial, and the like. In the lowest and highest organisms the injurious condition acts as a stimulus to produce many movements, subjecting the organism to various conditions, one of which is selected.

In connection with this method of behavior three questions arise, which are fundamental for the theory of regulation. First, How is it determined what shall cause the changes in behavior that result in new conditions? Or why does the organism change its behavior under certain conditions, not under others? Second, How does it happen that such movements are produced as result in more favorable conditions? Third, How is the more favorable condition selected; what is this selection and what does it imply? Our first and third questions may indeed be condensed into one, which involves the essence of the regulatory process: Why does the organism choose certain conditions and reject others? This selection of the favorable conditions and rejection of the unfavorable ones presented by the movements is perhaps the fundamental point in regulation.

It is often maintained that this selection is precisely personal or conscious choice, and that behavior cannot be explained without this factor. Personal choice it evidently is, and in man it is often conscious choice; ' whether it is conscious in other animals we do not know. But in any case this does not remove it from the necessity for analysis. Whether conscious or unconscious, choice must be determined in some way, and it is the province of science to inquire as to how this determination occurs. To say that rejection is due to pain, acceptance to pleasure, or to other conscious states, does not help us, for we are then forced to inquire 
why pain occurs under certain circumstances, pleasure under others. Surely this is not a haphazard matter! There must be some difference in the conditions to induce these differences in conscious states (if they exist) and at the same time to determine the differences in behavior. We are therefore thrown back upon the objective processes occurring. Why are certain conditions accepted, others rejected? This is essentially what has often been called the pleasure-pain problem.

Such facts as are set forth in the preceding paper give us a basis for an objective answer to this question. Organisms are not static structures; processes of complicated character are in continual progress within them. Among these the processes of metabolism are most prominent. Ostwald ('O2) has emphasized the point that one of the chief characteristics of living matter is the fact that processes are occurring with much energy within it. The organism is a complex of processes. In the preceding paper we have seen that the reactions of organisms to external agents depend largely on the relation of the action of these agents to the internal processes.

Let us examine certain cases of this dependence in the simplest organisms-bacteria and protozoa. The green Paramoecium bursaria requires oxygen in its metabolic processes. While swimming about it comes to a region where oxygen is lacking. It reacts by turning away and going in some other direction. The white Paramocium caudatum does the same, and so also do many bacteria. All require oxygen in their metabolic processes; lack of oxygen interferes with these processes, and they react to such a lack by changing their movement and going elsewhere. But there are some bacteria that do not require oxygen in their metabolic processes. When these come to a region lacking oxygen they do not react, but keep on and enter this region. In many of these anaërobic bacteria oxygen is known actually to interfere with the physiological processes. When these bacteria come to a region containing oxygen, they change their movement and go elsewhere. In Paramocia and the bacteria that require oxygen, this does not occur (unless the amount of oxygen rises above the optimum). In all these cases, whenever there is interference with the metabolic processes, the organism reacts by turning away, otherwise it does not. In the reactions of these creatures with reference to light and darkness we see the same thing. In the 
green Paramœcium bursaria, in Euglena, and many other green infusoria, light assists the metabolic processes, while lack of light interferes with them, and the same is true of the so-called purple bacteria. All of these organisms react on coming to a region of darkness by turning away and going elsewhere. In the colorless Paramœcium caudatum and in the colorless bacteria ordinary light does not affect the metabolic processes, so that there is no interference with these processes in darkness, and we find that they do not react on reaching a dark region, but enter it readily. For all these organisms, colored and uncolored, light may be made so intense that it does interfere with the physiological processes, as is shown by the fact that the processes stop, the organisms dying. To such light all react by turning away-including even the colorless Paramœcium caudatum. We find in all of these cases that the animal reacts by turning away when there is interference with the physiological processes, and does not so react unless there is such interference.

In some cases the relation between behavior and the effect of an agent on the physiological processes is marvelously precise. Thus, Engelmann ('82) proved that in Bacterium (Chromatium) photometricum the ultra red and the yellow-orange rays are those which most favor metabolism (assimilation of carbon dioxid, etc.). When a microspectrum is thrown on a preparation of these bacteria, they are found to react in such a way as to collect in precisely the ultra red and the yellow-orange. The reaction consists in a change of behavior - a reversal of movement-at the moment of passing from the ultra red or the yellow-orange to other parts of the spectrum, while passing in the opposite direction produces no such effect. Bacteria are not in nature subjected to spectral colors in bands, so that there has been no opportunity for the production of this correspondence between behavior and favoring conditions through the selection of varying individuals.

What is the explanation of these facts? Why does the infusorian or the bacterium shrink back from darkness or the region containing no oxygen? As a matter of fact, it requires the light or the oxygen for the continuance of its metabolic processes, and it does not shrink back from a region lacking them unless it does need them. But we have no reason to attribute to the bacterium any knowledge or idea of that relation. We do not need any 
purpose or idea in the mind of the organism, or any "psychoid" or entelechy to account for the change of behavior, for an adequate objective cause exists. We know experimentally that the darkness or the lack of oxygen interferes with the metabolic processes. This very interference is then evidently the cause of the change of behavior. When anything interferes with the internal processes, running with much energy, the energy overflows in other directions, resulting in changes in behavior. This statement is a mere generalized formulation of the facts determined by observation and experiment in the most diverse organisms.

Internal as well as external interference may cause the changes of behavior. If oxygen or other material for metabolism is lacking to such an extent as to interfere with the metabolic processes, the organism changes its behavior. In the sea anemones, as we have seen in the preceding paper, this condition induces the animal to change its position and start off on a laborious tour of exploration. The initiation of changes in movement through internal conditions gives the basis for the reactions which we call positive, as we shall see.

The answer to our first question is then as follows: The organism changes its behavior as a result of interference with its physiological processes.

Our second question was: How does it happen that such movements are produced as bring about more favorable conditions? This question we have already answered, so far as many lower organisms are concerned, in our general statement on page 474. The organism does not go straight for a final end. It merely acts-in all sorts of ways possible to it--resulting in repeated changes in the conditions. In this way a condition is after a time reached that relieves the interference with the internal processes.

The nature of the changes in behavior produced-the movements that occur in any given organism-depend on what may be called the "action system" of the organism. The animal, in other words, performs the movements that it is accustomed to perform, as determined by its structure and its past history. The essential fact is that interference with the internal processes causes a change in behavior. The mere fact of a change under these conditions tends in itself to be regulatory. The original behavior has brought on the interfering conditions, hence the 
best thing to do is to change this behavior. If the unfavorable condition still continues the behavior is changed again; this being continued, the organism is bound to escape from the interfering condition if it is possible to do so. In some cases the movements produced are, when considered by themselves, of a rather uniform character, yet are of such a nature as to subject the animal to many changes of the environmental conditions. This is the case for example in the reactions of such infusoria as Paramœcium, where the character of the movement is determined partly by structure, yet involves a continued change of relation to the outer conditions. In other cases the movements themselves are varied in character, the organism first reacts in one way, then in another, running through a whole series of activities, till one results in ridding the organism of the stimulating condition. This is the method of behavior seen in Stentor and in most higher organisms.

Our third question was: How does the organism select the more favorable condition thus reached? This question now answers itself. It was interference with the physiological processes that caused the changes in behavior. As soon, therefore, as this interference ceases, there is no further cause for change. The organism selects and retains the favorable condition reached merely by ceasing to change its behavior when interference ceases. This process is seen clearly in the behavior of such infusoria as Paramcecium.

It is perhaps fairly evident how reaction on the plan just described may result in the avoidance or rejection of sources of interfering stimuli; in other words, in the production of negative reactions. The miatter of positive reactions should perhaps receive further elucidation.

In conditions that are completely favorable-so that all the life processes are taking place without lack or hindrance-there is no need, from the standpoint of regulation, for a change in behaviorfor definite reactions of any sort The most natural behavior on reaching such conditions, and that which is actually found as a rule among lower organisms, is a continuation of the activities already in progress. These activities have resulted in the favorable conditions, and there is no cause for a change. This we find exemplified in infusoria, bacteria, rotifers, and many other organisms, under most classes of conditions. A change in behavior takes place only when the activities tend to remove the 
organism from the favorable conditions; in other words, to produce interference with the life processes. Unfavorable stimuli, in these organisms, cause a change in behavior; favorable stimuli cause none. It is perhaps a general rule in organisms, high or low, that continued completely favorable conditions do not lead to definite reactions. Of course while the external conditions remain the same, the internal processes may change in such a way that these conditions are no longer favorable, and now the behavior may change. This frequently happens.

When the organism is not completely enveloped by favorable conditions, but is on the boundary, if we may so express it, between favorable and unfavorable ones, there is often a definite change in the behavior leading toward the favorable conditions-a positive reaction. To understand such reactions, we may start from the fact, already mentioned, that unfavorable internal conditions (as well as external ones) cause a change of behavior. It is a general fact, for example, that the animal whose metabolic processes suffer interference from lack of material-the hungry animal--sets in operation trains of activity differing from the usual ones. Interference with respiration, or an increase in temperature above that favorable for the physiological processes, has similar effects. This is indeed a general rule for all internal changes interfering with the usual physiological processes.

But the activities thus induced are in themselves undirected, save by structural conditions. There is nothing in the cause producing them to direct them with reference to external things. Let us suppose, however, that certain of these movements lead to a condition which relieves the interference with the internal processes. The cause for a change of behavior is now removed, hence the organism continues its present movement. But perhaps later-sometimes at the very next instant-this same movement may tend to remove the organism from the favorable conditionas when a Paramœcium in a heated preparation passes across a small area of water cooled to the optimum, and reaches the opposite side, or when a hungry organism comes in contact with food, which will be lost if there is further movement. Thereupon the cause for a change-interference with the life processes-is again set in operation, and the present movement is changed. Thus the animal changes all behavior that leads away from the favorable condition, and continues that which tends to retain it, so that we 
get what we call a positive reaction. The change of behavior is due in each case primarily to the unfavorable stimulation, internal or external. This style of behavior is seen with diagrammatic clearness in the free swimming infusoria. These animals continue their movements as long as they lead to favorable conditions, changing at once such movements as lead away. They thus retain favorable conditions by avoiding unfavorable ones; the positive reaction is seen to be, in a sense, a secondary result of negative ones.

We have a similar condition of affairs in the taking of food by Amœba. The animal moves forward with broad front, and comes in contact at a certain point on this front with a food body. Part of its movement is taking it away from the food, part toward the food. On coming in contact, all movement which takes it away is changed, only that being continued which keeps the animal in contact with the food. We have here then the same condition of affairs as in the infusoria-the selection of certain conditions through the rejection of all others.

This is perhaps the fundamental condition of affairs for organisms in general. In higher animals the positive, as well as the negative, reactions, have become complicated through the influences to be brought out later, so that this primitive condition is not evident. But the essential point is that unfavorable conditions are rejected as a result of the fact that they produce changes in behavior, and this results in the attainment and retention of favorable conditions. In negative reactions it is the new unfavorable external condition that is rejected, retaining the old favorable internal condition. In positive reactions it is the old unfavorable internal conditions that are rejected, retaining the new favorable external conditions. In both cases the impulse to change of movement comes from interference with the physiological processes - external interference in negative reactions, internal interference in positive reactions.

To sum up, in the lowest organisms we find individual adjustment or regulation on the basis of the three following facts:

I. Definite internal processes are occurring in organisms.

2. Interference with these processes causes a change of behavior and varied movements, subjecting the organism to many different conditions.

3. One of these conditions relieves the interference with the 
internal processes, so that the changes in behavior cease, and the relieving condition is thus retained.

It is clear that regulation taking place in this way does not require that the end or purpose of the action shall function in any way as part of its cause, as some vitalistic theories hold. There is no objective evidence that a final aim is guiding the organism. None of the factors above mentioned appear to include anything differing in essential principle from such laws of causality as we find in the inorganic world.

Now an additional factor enters the problem. By the process which we have just considered, the organism reaches in time a movement that brings relief from the interfering conditions. This relieving response becomes fixed through the operation of a certain law which appears to hold throughout organic activities. This law may be stated as follows: An action performed or a physiological state reached, is performed or reached more readily after one or more repetitions, so that in time it becomes "habitual." The statement of this law just given is in reality not adequate, and it may be well to dwell upon it a moment, developing it farther, and pointing out some of the phenomena in which it is expressed. In previous papers, including the one immediately preceding the present, I have pointed out the fact that the behavior and reactions of an organism depend largely on "physiological states;" the same point has recently been emphasized by Bohn ('05). We may distinguish at least two great classes of physiological states-those depending on the metabolic processes of the organism, treated in detail in the preceding paper, and those otherwise determined. The physiological states of organisms change in accordance with certain laws. The changes in the metabolic states of course depend on the laws of metabolism. In the physiological states not directly dependent upon metabolism, but rather upon stimulation and upon the activity of the organism, such as are found in Stentor and Planaria (see Jennings, 'o4), we find certain fairly well defined laws of change that are of a peculiar character.

In the organisms just mentioned, and in many others, the following phenomena have been found. Under certain external conditions the organism reacts in a certain way. These conditions continuing, the organism changes its first reaction for a second, and then perhaps for a third and fourth. Later the same external conditions recur, and now the organism at once 
responds, not by its first reaction, but by the final one. This is illustrated for unicellular organisms by the case of Stentor, for higher metazoa by the behavior of certain crustacea, as described by Yerkes ('O2) and Spaulding ('O4). There are certain differences between the two cases, but they are not essential for our present purpose.

How does this condition of affairs come about? As we have set forth in previous papers ('O4), the different methods of reaction under the same external conditions must be due to different physiological states of the organism. The "physiological state" is evidently to be looked upon as a dynamic condition, not as a static one; it is a certain way in which the bodily processes are occurring; it tends directly to the production of some change. In this respect the "law of dynamogenesis," propounded for ideas of movement in man, applies to it (see Baldwin, '97, p. 167); ideas must indeed be considered, so far as their objective accompaniments are concerned, as certain physiological states in higher organisms. The changes toward which physiological states tend are of two kinds. First, the physiological state, like the idea, tends to produce movement. This movement often results in such a change of condition as destroys the physiological state producing it. But in case it does not, then the second tendency of the physiological state shows itself. It tends to resolve itself into another and different state. State I passes to state 2, and this again to state 3. This tendency shows itself even when the external conditions remain uniform.

In this second tendency there manifests itself the important law of which we have spoken above. When a certain physiological state has been resolved, through the action of an external agent, or otherwise, into a second physiological state, this resolution becomes easier, so that in the course of time it takes place more quickly, and even spontaneously.

This may be illustrated from the behavior of the unicellular organism Stentor, as described in previous papers by the present writer ('O2 and 'O4), as follows: When the animal is stimulated by the flood of carmin grains (or in any other way), this produces immediately a certain physiological state corresponding to that accompanying a sensation in ourselves. This state we may designate A. It at first produces no reaction. As the carmin continues or is repeated, this state A passes to a second state 
$\mathrm{B}$, producing a bending to one side. After several repetitions of the stimulus, the state $\mathrm{B}$ passes to the state $\mathrm{C}$, producing a reversal of the cilia, and this finally passes to $\mathrm{D}$, resulting in a contraction of the body. Each state must of course be different from the preceding one, because it produces a different result. The course of the changes in physiological states may then be represented as follows:

$$
\mathrm{A} \longrightarrow \mathrm{B} \longrightarrow \mathrm{C} \longrightarrow \mathrm{D}
$$

Now we find that after many repetitions of the stimulation the animal contracts at once as soon as the carmine comes in contact with it. In other words, the first condition A (direct result of contact) passes at once to the state $\mathrm{D}$, and this results in immediate contraction:

$$
\mathrm{A} \longrightarrow \mathrm{D}
$$

It seems probable that the same series occurs as before, save that $\mathrm{B}$ and $\mathrm{C}$ are now passed rapidly and in a modified way, so that they do not result in a reaction, but are resolved directly into D. The process would then be represented as follows:

$$
\mathrm{A} \longrightarrow \mathrm{B}^{\prime} \longrightarrow \mathrm{C}^{\prime} \longrightarrow \mathrm{D}
$$

But whatever the intermediate conditions, it is clear that after the state A has become resolved, through pressure of external conditions, into the state $\mathrm{D}$, this resolution takes place more readily, occurring at once after state $A$ is reached.

The same law is illustrated in the experiments of Yerkes and Spaulding on association in crabs. In the experiments of Spaulding ('O4) with hermit crabs, the introduction of the dark screen into the aquarium, and the diffusion of the juices of the fish, cause the animals to move about. In so doing they reach the dark screen, which induces, let us say, the physiological condition A. This leads to no special reaction. But this is followed regularly by contact with food, inducing the physiological state $\mathrm{B}$, which is concomitant with a positive reaction. The physiological state A is thus regularly resolved into the state $B$. In the course of time this resolution becomes automatic, so that as soon as the state $\mathrm{A}$ is reached, it passes to $\mathrm{B}$. The positive reaction concomitant with $\mathrm{B}$ is therefore given even though the original cause of $\mathrm{B}$ is absent. The actual number of physiological states which could be dis- 
Metbod of Regulation in Bebavior and in Other Fields. 485

tinguished is of course greater than what we have set forth, but this does not alter the principle involved.

The law which we have just brought out may then be summed up as follows:

The resolution of one physiological state into another becomes easier and more rapid after it has taken place one or more times. Hence the behavior primarily characteristic for the second state comes to follow immediately upon the first.

The operations of this law are seen on a vast scale in higher organisms, where they constitute what we commonly call memory, association, habit, and the basis of intelligence. It has been shown to hold in a number of lower organisms, though in these the manifestations of this law are comparatively little known. Yerkes and Spaulding have demonstrated its applicability to Crustacea. The low acelous flatworm Convoluta evidently shows it clearly, since as we have seen in the preceding paper, it forms definite habits. It has even been demonstrated, as we have seen, in the protozoa, particularly Stentor and Vorticella. According to Hodge and Aikins ('95) a method of reacting thus developed lasted in Vorticella as long as five hours. In view of these facts, it is probable that the law is a general one and that it will be demonstrated in some form for other lower organisms. There seems to be no theoretical reason for supposing it to be limited to higher animals. The paucity of experiments fitted to test it is amply sufficient to account for the very slight knowledge we have of it in lower organisms.

To return then to the thread of our discussion: In virtue of this law of the readier resolution of physiological states after repetition, the final reaction of a trial series, relieving the organism of the interference with its physiological processes, is later reached more readily than at first, and in time becomes the immediate reaction to the interfering condition. Thus the change of behavior induced by interference of a certain sort has come to be of a perfectly definite character, and all trial movements are omitted.

It is in this second stage of the process, when the relieving response has become set through the law above discussed, that an end or purpose seems to dominate the behavior. This end or purpose of course actually exists, as a subjective state called an idea, in man. Whether any such subjective state exists in the 
lower organism that has gone through the process just sketched we of course do not know. But some objective phenomenon, as a transient physiological state, corresponding to the objective physiological accompaniment of the idea in man, would seem to be required in the lower organism. The behavior in this stage is that which, in its higher reaches at least, has been called intelligent.

But so far as the objective occurrences are concerned there would seem to be nothing in even this later stage of behavior involving anything different in principle from what we find in the inorganic world. The only additional feature is this law of the readier attainment of a certain state or action after repetition. We have not attempted to state this law in an entirely adequate manner, but there would seem to be nothing implied by it that is specifically vital, in the sense that it differs in essential principle from what we find in the laws of causality as applied to the inorganic world. It certainly by no means requires in itself the action of any "final cause"- that is, of an entity that is at the same time purpose and cause. On the other hand, it undoubtedly does produce that type of behavior which has given rise to the conception of the purpose acting as cause. This conception is in itself of course a correct one, so far as we mean by a purpose an actual physiological state of the organism, determining behavior in the same manner as other factors determine it.

That regulation takes place in the behavior of many animals in the manner above sketched seems to the writer an established fact, and it appears to be perhaps the only clearly intelligible way in which regulatory behavior could be developed in a given individual.

But we are of course confronted with the fact that many individuals are provided at birth with definitely regulatory methods of reaction to certain stimuli. In these cases the animal is not compelled to go through the process of performing trial movements, with subsequent fixation of the successful movement. How are such cases to be accounted for?

If the regulatory methods of reaction acquired through the process sketched in the preceding paragraphs could be inherited, there would of course be no difficulty in accounting for such congenital regulatory reactions, or habits. It is perhaps not going too far to say that this possibility is not yet out of court, though 
opinion at present seems to be generally against it. Yet Semon ('04) in his recent valuable monograph on the phenomena allied to memory and habit, maintains the affirmative view, and presents evidence in favor of it. We are in the beginning of the study of such problems, and it can hardly be said that experiments of sufficient duration and precision have yet been tried to really test the matter. If the inheritance of regulatory reactions acquired after trial should be demonstrated, the process sketched above would give us a satisfactory general method for the development of regulatory behavior, in the race as well as in the individual. In the protozoa this difficulty of course does not exist; the acquirements of individuals may remain as acquirements of the race.

If such inheritance does not occur, then the existence of congenital definite regulatory reactions would seem to be explicable only on the basis of the selection of individuals having varying methods of reaction, unless we are to adopt the theories of vitalism. In the method we have sketched above, a certain reaction that is regulatory is selected, through the operation of physiological laws, from among many performed by the same individual. In what is called natural selection, the same reaction is selected from among many performed by different individuals-in both cases because it is regulatory-because it assists the physiological processes of the organism. The two factors must work in the same direction. "Intelligence" and natural selection are two analogous methods of selecting the adaptive reactions from among the possible ones; they must then work together, as Baldwin ('O2) has so well pointed out. Which of the two factors is the essential one in producing congenital adaptive reactions we shall of course not attempt to decide, since no one knows.

We often find in organisms behavior that is not regulatory, or that is regulatory only in a very imperfect way. How are we to account for this? Without going into details, it is evident that there exist at least three general conditions that may result in non-regulatory behavior. First, the organism is formed of substance that is subject to the ordinary laws of physics and chemistry. Various physical and chemical agents may act directly upon this substance, producing results that are not regulatory. The fact that the relation of external processes to internal ones is one of the chief determining factors in producing reactions, of 
course does not exclude the possibility of the direct action of agents on the body substance. The operation of intense physical and chemical agents may injure or destroy the substance of which the organism is composed, and with it the organism, in spite of any reaction the organism can give. Second, the organism can perform only those movements which its structure permits. Often none of these movements can relieve the existing interference with the physiological processes. Then the organism can only try them, without regulatory results, and die. Examples of this are seen in the behavior of Paramocium, or of Planaria when placed in heated water. Both animals perform practically all the reactions of which they are capable, before they succumb. Third, certain responses may become fixed, in the way sketched above, because under usual conditions they relieve the organism. Now if the conditions change, the organism can respond at first only by this fixed reaction, and if this does not relieve, the animal may be destroyed before a new regulatory reaction can be developed. This condition of affairs is widespread among animals.

All together, the regulatory character of behavior as found in many animals seems perhaps intelligible in a perfectly natural, directly causal way, on the basis of the principles brought out above. We may summarize these principles as (I) the selection by varied movements of conditions not interfering with the physiological processes of the organism; (2) the fixation of the movements by which the selected conditions were reached, by the law of the readier resolution of physiological states after repetition. Neither of these principles seem to contain anything specifically vitalistic, or opposed in principle to what we find in the inorganic world.

Is it possible that regulation is based on similar principles in other fields than behavior? Bodily movement is only one of the many kinds of activity that may vary, and variations of any of the organic activities may impede or assist the physiological processes of the organism. Is it possible that interference with the physiological processes may induce changes in other activities-in chemical processes, in growth, and the like-and that one of these activities is selected, as in behavior, through the fact that it relieves the interference that caused the changes?

There is some evidence for this possibility. Let us look for 
example at regulative changes in the chemical activity of the organism, such as we see in acclimatization to poisons, in responses to changes in temperature, or in the adaptation of the digestive juices to the food. What is the material from which the regulative changes may be selected? One of the general results of modern physical chemistry is expressed by Ostwald ('02, p. 366) as follows: "In a given chemical structure all processes that are possible, are really taking place, and they lead to the formation of all substances that can occur at all." Some of these processes are taking place so slowly that they escape usual observation; we notice only those that are conspicuous. But in its enzyms the body possesses the means of hastening any of these processes and delaying others, so that the general character of the action shall be determined by the more rapid process. Such enzyms are usually present in the body in inactive forms (zymogens), which may be transformed into active enzyms by slight chemical changes, thus altering fundamentally the course of the chemical processes in the organism.

It is evident that the organism has presented to it, by the condition just sketched, unlimited possibilities for the selection of different chemical processes. The body is a great mass of the most varied chemicals, and in this mass thousands of chemical processes in every direction-all those indeed that are possibleare occurring at all times. There is then no difficulty as to the sufficiency of the material presented for selection, if some means may be found for selecting it. The process which will relieve any unfavorable condition, if any such process is possible, is actually occurring in an infinitesimal way, and needs only to be hastened.

Further, it is known that interference with the physiological processes does result in many changes in the internal activities of the organism, as well as in its external movements. Intense injurious stimulation causes not only "excess" movements of the body as a whole, but induces marked changes in circulation, in respiration, in temperature, in digestive processes, in excretion, and in other ways. Such marked internal changes involve, and indeed are constituted by, alterations of profound character in the chemical processes of the organism. These chemical changes are sometimes demonstrated by the production of new chemical substances under such circumstances. Furthermore, it is clear that the internal changes due to interference with the 
physiological processes are not stereotyped in character, but varied. Under violent injurious stimulation respiration may become for a time rapid, then is almost suspended. The heart for a time beats furiously, then feebly, and there is similar variation in other internal symptoms.

Thus it seems clear that interference with the life processes does induce varied activities in other ways than in bodily movements, and that among these are varied chemical processes. There is then presented opportunity for regulation to occur in the same way as in behavior. Certain of the processes occurring relieve the disturbance of the physiological functions. There results then a cessation of the changes. In other words, a certain process or condition is selected through the fact that it does relieve.

There is much evidence that the law of the readier resolution of physiological states after repetition applies to other bodily processes as well as to behavior. The much readier induction of digestive trouble by a small quantity of a certain food, after a large quantity has once induced it is perhaps an example; many better ones are given by Semon ('05). If the analogy with behavior holds in this respect, there will be present at a later period certain fixed methods of chemical response, by which the organism reacts to certain sorts of stimulation-as by the production of a definite antitoxin when a certain poison is introduced. Definite organs or organisms will have left open to them only certain limited possibilities of variation-due to the development of something corresponding to the "action system" in behavior. Thus, in the pancreas there will not exist unlimited possibilities as to the chemical changes that may easily occur. Its "action system" will be limited perhaps to the production of varied quantities of certain enzyms-amylopsin, trypsin, etc. The proper selection of these few possibilities will then occur by the general method sketched. When digestion is disturbed by food that is not well digested, variations in the production of the different enzyms will be set in train, and one of these will in time relieve the difficulty, through the more complete digestion of the food. Thereupon the variations will cease, since their cause has disappeared. By still more complete fixation of the chemical response, through the law of the readier resolution of physiological states after repetition, an organ or organism may largely lose its power of varying its chemical behavior, and thus be unable to 
meet new conditions in a regulative way. A condition comparable to the establishment of a fixed reflex in behavior will result.

It is perhaps more difficult to apply the method of regulation above set forth to processes of growth and regeneration. Yet there is no logical difficulty in the way. The only question would be that of fact-whether the varied growth processes necessary do primitivelyoccur, under conditions that interfere with the physiological processes. When a wound is made or an organ removed, is the growth process which follows always of a certain stereotyped character, or are there variations? It is, of course, well known that the latter is often the case. In the regeneration of the earthworm, Morgan ('97) finds great variation; he says that in trying many experiments, one finds that what ninety-nine worms cannot do in the way of regeneration, the one hundredth can. The very great variations in the results of operations on eggs and young stages of animals is well known. Removal of an organ is known to produce great disturbance of most of the processes in the organism, and among others, in the process of growth.

It appears then not impossible that in growth processes regulation may be brought about in accordance with the same principles as in behavior. A disturbance of the physiological processes results in varied growth activities. Some of these relieve the disturbance; the variations then cease, and these processes are continued. In any given highly organized animal or plant the different possibilities of growth will have become practically much limited, and it is only from this limited number of possibilities that selections can be made. In some cases, by the fixation of certain processes through the analogue of the law of the readier resolution of physiological states, the organism or a certain part thereof will have lost the power of responding to injury save in one definite way. Under new conditions this one way may not be regulatory, yet it may be the only response possible. This may result in the formation under certain conditions of such things as heteromorphic structures-a tail in place of a head, or the like, from a part of the body that is accustomed (in normal development) to produce such an organ. This would again correspond to the production of a fixed reflex action in behavior, even under circumstances where this action is not regulatory.

It appears to the writer that the method of form regulation recently developed in a most suggestive paper by Holmes ('04) 
is in essential agreement with the general method of regulation here set forth, and may be considered a working out of the details of the way in which growth regulation would probably take place along such lines.

It may be noted that regulation in the manner we have set forth is what, in the behavior of higher organisms, at least, is called intelligence. If the same method of regulation is found in other fields, there is no reason for refusing to compare the action to intelligence. Comparison of the regulatory processes that are shown in internal physiological changes and in regeneration to intelligence seems to be looked upon sometimes as heretical and unscientific. Yet intelligence is a name applied to processes that actually exist in the regulation of movements, and there is, a priori, no reason why similar processes should not occur in regulation in other fields. When we analyze regulation objectively, there seems indeed reason to think that the processes are of the same character in behavior as elsewhere. If the term intelligence be reserved for the subjective accompaniments of such regulation, then of course we have no direct knowledge of its existence in any of the fields of regulation outside of the self, and in the self perhaps only in behavior. But in a purely objective consideration there seems no reason to suppose that regulation in behavior (intelligence) is of a fundamentally different character from regulation elsewhere.

It is perhaps hardly necessary to point out the relation of the method of regulation in behavior here discussed to the process of "selection of overproduced movements," so ably set forth in Baldwin's well-known works ('97, 'O2). The account here given is based on this same process, but differs in a number of points which seem to the writer of fundamental significance for a proper understanding of the method of regulation. Baldwin has likewise made some suggestion as to the possibility of extending this point of view to other fields (Baldwin '02).

We may make a general statement of the features in the method of regulation set forth in this paper, as follows: The organism is primarily activity. It is the seat of many processes, of chemical change, movement, and growth; these are proceeding with a certain amount of energy. These processes depend for their unimpeded course on one another and on the relations to the environment which the processes themselves largely bring about. 
When any of the processes are blocked the energy overflows in other directions, producing varied changes in chemical processes, movement and growth. Some of the conditions reached through these changes relieve the interference that was the cause of the changes. Thereupon the changes cease, since their cause has disappeared; the relieving condition is therefore maintained. After repetition of this course of events, the change which leads to relief is reached more directly, as a result of the law of the readier resolution of physiological states after repetition. Thus are produced finally the stereotyped and under certain conditions nonregulatory changes sometimes resulting from stimulation.

This method of regulation is clearly seen in behavior, where its operation is, in the later stages, what is called intelligence. Its application to chemical and form regulation is at present hypothetical, but appears probable.

\section{LITERATURE CITED.}

Baldwin, J. Mark, '97.--Mental Development in the Child and in the Race. Methods and Processes. Second ed., 496 pages. New York. '02.-Development and Evolution. 395 pages. New York.

BoHN, G., '05.-Attractions et Oscillations des animaux marins sous l'influence de la lumière. Institut Général Psychologique, Mémoires, i, i ro pages.

Engelmann, Th. W., '82.--Bacterium photometricum. Ein Beitrag zur vergleichenden Physiologie des Licht- und Farbensinnes. Arch. f. d. ges. Physiol., Bd. xxx, S. 95-124.

Hodge, C. F., And Aikins, H. A., '95--The Daily Life of a Protozoan: a Study in Comparative Psycho-physiology. Amer. Journ. Psychol., vol. vi, pp. 524-533.

Holmes, S. J., '04.-The Problem of Form Regulation. Arch. f. Entw.-mech., Bd. xviii, S. 265-305.

Jennings, H. S., 'o2.- Studies on Reactions to Stimuli in Unicellular Organisms. IX. On the Behavior of Fixed Infusoria (Stentor and Vorticella), with Special Reference to the Modifiability of Protozoan Reactions. Amer. Journ. Physiol., vol. viii, pp. 23-60.

'04.-Physiological States as Determining Factors in the Behavior of Lower Organisms. Contributions to the Study of the Behavior of the Lower Organisms, fifth paper, pp. 109-127. Carnegie Institution of Washington, Publ. r6. 
Morgan, T. H., '97.-Regeneration in Allolobophora fotida. Arch. f. Entw.mech., Bd. v, S. 570-586.

Ostwald, W., '02.-Vorlesungen über Naturphilosophie. 457 pages. Leipzig. Semon, R., '04.-Die Mneme als erhaltendes Prinzip im Wechsel des organischen Geschehens. 353 pages. Leipzig.

Spaulding, E. G., '04.-An Establishment of Association in Hermit Crabs, Eupagurus longicarpus. Journ. Comp. Neurol. and Psychol., vol. xiv, pp. 49-6I.

Yerkes, R. M., '02.-Habit Formation in the Green Crab, Carcinus granulatus. Biol. Bull., vol. iii, pp. 241-244. 